\title{
CARACTERÍSTICAS CLÍNICAS DE PACIENTES SUBMETIDOS A INTERVENÇÃO CORONÁRIA PERCUTÂNEA NOS ANOS DE 2014 A 2019
}

\section{CLINICAL CHARACTERISTICS OF PATIENTS UNDERGOING PERCUTANEOUS CORONARY INTERVENTION IN THE YEARS 2014 TO 2019}

\section{Letice Dalla Lana ${ }^{1} *$ Liliane Gonçalves Oliveira ${ }^{2} *$ Joseane Trindade Nogueira $^{3} *$ Jamille Louise $^{6}$ Bortoni de Oliveira $^{4} *$ Cenir Gonçalves Tier ${ }^{5}$ Ana Karina Silva da Rocha Tanaka ${ }^{6}$ Paulo Emilio Botura Ferreira ${ }^{7}$}

\section{RESUMO}

Objetivo: Identificar as características clínicas de pacientes submetidos a intervenção coronária percutânea (ICP) entre os anos de 2014 a 2019. Métodos: estudo transversal retrospectivo, com abordagem quantitativa. Incluíram-se prontuários de pacientes adultos, de ambos os sexos, com idade igual ou superior a 18 anos, submetidos ao procedimento de ICP diagnóstico e/ou terapêutico em um serviço de hemodinâmica localizado na Fronteira-Oeste do Rio Grande do Sul. A análise deu-se por meio de estatística descritiva simples. Resultados: Dos 344 prontuários de pacientes submetidos a ICP, distribuídos nos seis anos, verificou-se que a amostra ficou composta de $(\mathrm{n}=225 ; 65,4 \%)$, sexo masculino com média de idade de 62,98 anos $(\mathrm{Dp} \pm 10,03)$. No que se refere às características clínicas, $110(31,97 \%)$ pacientes internaram por angina pectoris seguido de 107 (31,10\%) para Infarto Agudo do Miocárdio (IAM). O principal sintoma relatado pelos pacientes na chegada ao atendimento de saúde foi a angina $(n=240 ; 69,76 \%)$. Um pouco mais da metade $(n=200 ; 58,13 \%)$ foi submetida apenas à angioplastia com a colocação de um, dois ou três stents. $\mathrm{O}$ desfecho clínico foi alta hospitalar $(\mathrm{n}=331 ; 96,22 \%)$. Conclusão: O conhecimento das características dos pacientes submetidos a ICP torna-se fundamental para otimizar o processo de trabalho e de cuidado possibilitando, ao enfermeiro, implementar intervenções de cuidado de modo articulado com a rede de atenção à saúde.

Palavras-chave: Hemodinâmica; Intervenção Coronária Percutânea; Enfermagem Cardiovascular; Angioplastia; Perfil de Saúde.

\section{ABSTRACT}

Objective: To identify the clinical characteristics of patients undergoing percutaneous coronary intervention (PCI) between 2014 and 2019. Methods: retrospective cross-sectional study with a quantitative approach. We will include medical records of adult patients, of both sexes, with a diet of 18 years or more, undergoing diagnostic and/or therapeutic PCI procedures in a hemodynamic service located in the West Border of Rio Grande do Sul. simple descriptives. Results: Two 344 medical records of patients undergoing PCI, distributed over six years, it was found that it was composed of $(n=225 ; 65.4 \%)$, male with a mean age of 62.98 years $(\mathrm{SD} \pm 10.03)$. Not referring to clinical characteristics, $110(31.97 \%)$ patients were hospitalized for angina pectoris, followed by 107 (31.10\%) for Acute Myocardial Infarction (AMI). The main symptom reported in patients after healthcare or care for angina $(n=240 ; 69.76 \%)$. A little more than the target $(n=$ $200 ; 58.13 \%$ ), only angioplasty was performed with the placement of one, two or three stents. Either clinical failure or hospital discharge $(\mathrm{n}=331 ; 96.22 \%)$. Conclusion: The knowledge of the characteristics of two patients undergoing PCI is essential to optimize the work and care process, enabling, as a nurse, to implement assistance interventions in an articulated manner as a health care network.

Keywords: Hemodynamics; Percutaneous Coronary Intervention; Cardiovascular Nursing; Angioplasty; Health Profile.

\footnotetext{
${ }^{1}$ Enfermeira, Mestre em Geriatria e Gerontologia Biomédica, Doutora em Enfermagem. Docente da Graduação e Pós-Graduação na Universidade Federal do Pampa (UNIPAMPA), Membro do Departamento Científico Enfermagem Gerontológica da Associação Brasileira de Enfermagem- RS. Uruguaiana, Brasil, ORCID: 0000-0002-9624-8152

${ }^{2}$ Enfermeira, Especialista em Cardiologia e Hemodinâmica; Hospital Santa Casa, Uruguaiana, Brasil, ORCID: 0000-0001-8179-4559

${ }^{3}$ Enfermeira do Hospital Santa Casa, Uruguaiana, Brasil, ORCID: 0000-0003-0406-6376

${ }^{4}$ Acadêmica de Enfermagem da Universidade Federal do Pampa (UNIPAMPA), Bolsista FAPERGS, Uruguaiana, Brasil, ORCID: 0000-0003-47262123

${ }^{5}$ Enfermeira, Mestre e Doutora em Enfermagem. Docente da Universidade Federal do Pampa (UNIPAMPA). Líder do Grupo de Estudos e Pesquisa sobre Envelhecimento Humano na Fronteira- GEPESH-FRON. ORCID: 0000-0003-1539-7816

${ }^{6}$ Enfermeira, Mestre e Doutora em Geriatria e Gerontologia Biomédica. Membro Departamento Científico Enfermagem Gerontológica da Associação Brasileira de Enfermagem e Membro pertencente a Diretoria da SOBENDE/RS (Sociedade Brasileira de Enfermagem Dermatológica). Docente da Universidade Federal do Rio Grande do Sul (UFRGS), Brasil, ORCID: 0000-0003-2488-3656.

7 Biólogo, Mestre, Doutor e Pós-Doutor em Ciências Biológicas. Docente da Graduação e Pós-Graduação na Universidade Federal do Pampa (UNIPAMPA), Uruguaiana, Brasil, ORCID: 0000-0003-1196-4668
} 


\section{INTRODUÇÃO}

As Doenças Cardiovasculares (DCV) são a primeira causa de mortes no Brasil ${ }^{(1)}$, sendo diagnosticadas e tratadas por meio de um procedimento minimamente invasivo, denominado respectivamente, como cateterismo cardíaco e angioplastia coronariana. O cateterismo tem finalidade de identificar e caracterizar os dados anatômicos e funcionais das possíveis lesões coronarianas $^{(2)}$. Enquanto que a angioplastia coronariana consiste na desobstrução de artérias através de um balão ou inserção de um ou mais stents no interior do vaso lesionado ${ }^{(3)}$.

$\mathrm{O}$ avanço tecnológico e científico disponibilizado nas unidades de hemodinâmica demandam de uma equipe profissional qualificada para garantir uma prática avançada à saúde. Entretanto, para se obter a qualidade e efetividade dos serviços é de extrema importância um enfermeiro líder, membro de uma equipe multidisciplinar, que viabilize recursos educacionais, tecnológicos e assistenciais para uma assistência integralizada e de qualidade ${ }^{(4)}$.

As competências do enfermeiro na hemodinâmica exigem habilidade, conhecimento e atitude aos quais conduziram ao julgamento clínico e pensamento crítico. Entretanto, para otimizar o processo de trabalho e cuidado, bem como favorecer ações de educação e atenção em saúde com vistas na prevenção de agravos à saúde, torna-se necessário conhecer as características dos pacientes submetidos aos procedimentos de diagnóstico e tratamento coronariano ${ }^{(5)}$.

Cabe ao enfermeiro na unidade de hemodinâmica desenvolver uma assistência sistematizada articulada nos períodos pré, trans e pós operatório do paciente submetido a Intervenção Coronária Percutânea $(\mathrm{ICP})^{(5)}$. Em adição, o levantamento precoce de fatores de risco na avaliação do enfermeiro conduzirá a práticas avançadas em saúde, minimizando complicações clínicas e emergenciais a curto, médio e longo prazo, seja na atenção primária, secundária e terciária à saúde ${ }^{(6)}$.

Neste contexto, a pesquisa sobre as características clínicas de pacientes submetidos à angioplastia e cateterismo no município, visa ampliar o conhecimento acerca dos cuidados pré e pós intervenção coronariana. Com base nisso, o objetivo do presente estudo foi identificar as características clínicas de pacientes submetidos a intervenção coronária percutânea (ICP) entre os anos de 2014 a 2019.

\section{MÉTODO}

Trata-se de um estudo retrospectivo, descritivo, transversal, de abordagem quantitativa, que foi realizado em um serviço de hemodinâmica de um hospital da Fronteira Oeste do Rio Grande do Sul, Brasil. Optou-se por este serviço, pois durante o período do estudo, não foram encontrados estudos 
similares e atuais sobre a caracterização dos pacientes atendidos na Rede de Atenção à Saúde da região. Ademais, optou-se por este serviço de hemodinâmica, visto que era referência ao município da $10^{\mathrm{a}}$ Coordenadoria de Saúde até o ano de 2018.

Os critérios de inclusão foram: prontuários físicos de pacientes adultos, de ambos os sexos, com idade igual ou superior a 18 anos, submetidos ao procedimento de ICP diagnóstico e/ou terapêutico entre os anos de 2014 e 2019. Optou-se pelo ano de 2014, tendo em vista a disponibilidade dos prontuários físicos no serviço de arquivo médico (SAME). Os critérios de exclusão foram: prontuários incompletos que não descreveram o tipo de intervenção cardíaca, a doença correlacionada com a necessidade de intervenção cardíaca e desfecho clínico em alta, transferência e óbito.

A coleta dos dados foi realizada nos meses de julho e agosto de 2020, por meio de um instrumento tipo checklist, previamente elaborado pelos autores mediante literatura relacionada.

A amostra foi de 344 prontuários distribuídos nos seis anos para coleta retrospectiva numa margem de confiança de $95 \%$ e erro de 5\%. Como a média de atendimento foi de 100 procedimentos por mês no serviço de hemodinâmica, calcula-se 9.600 procedimentos entre os 6 anos.

A análise foi por meio de estatística descritiva simples. As variáveis categóricas foram expressas com frequências absolutas e percentis e a variável contínua foi descrita pela mediana, quartis, média, desvio padrão e variância.

O projeto foi aprovado pelo Hospital, com assinatura do termo de Autorização de Instituição Co-participante e do Comitê de Ética em Pesquisa em Seres Humanos da Universidade Federal do Pampa- Campus Uruguaiana, sob parecer CAAE: 30498320.0.0000.5323.

\section{RESULTADOS}

A amostra do estudo foi de 344 prontuários de pacientes submetidos a ICP entre os anos de 2014 e 2019. Verificou-se que um pouco mais da metade da amostra era do sexo masculino $(\mathrm{n}=225 ; 65,4 \%)$ com média de idade de 62,98 anos ( $\mathrm{Dp} \pm 10,03)$, máxima de 89 anos e mínima de 36 anos. Quase metade dos pacientes eram casados $(\mathrm{n}=164 ; 48 \%)$ e possuíam o ensino fundamental $(\mathrm{n}=202 ; 59 \%)$. Majoritariamente, os pacientes são de cor branca $(n=281$; $81,68 \%)$. Na tabela 01 , apresenta-se os dados referentes ao perfil dos pacientes.

Tabela 01- Perfil dos pacientes submetidos a ICP. Uruguaiana, Brasil, 2021

\begin{tabular}{lll}
\hline Variável & $\mathrm{n}$ & $\%$ \\
\hline Sexo & & \\
\hline
\end{tabular}




\begin{tabular}{lll}
\hline Masculino & 225 & 65,4 \\
Feminino & 119 & 34,6 \\
Cor & & \\
Branca & 281 & 81,68 \\
Parda & 40 & 11,62 \\
Negra & 13 & 3,77 \\
Não registrado & 09 & 2,61 \\
Estado civil & & \\
Casado/União estável & 164 & 47,7 \\
Solteiro/Divorciado/Viúvo & 130 & 37,7 \\
Outros & 05 & 1,5 \\
Não registrado & 45 & 13,1 \\
Escolaridade* & & \\
Ensino Fundamental & 202 & 59 \\
Ensino Médio & 50 & 14,6 \\
Analfabeto & 16 & 4,7 \\
Ensino Superior & 06 & 1,8 \\
Não registrado & 68 & 19,9
\end{tabular}

* Incompleto ou Completo.

Fonte: Os autores

No que se refere às características clínicas dos pacientes, $(n=110 ; 31,97 \%)$ internaram por angina pectoris seguido de $(\mathrm{n}=107 ; 31,10 \%)$ para Infarto Agudo do Miocárdio (IAM). Apenas 60 prontuários $\quad(17,44 \%)$ apresentavam informações sobre a presença de duas ou mais doenças preexistentes relacionadas ao sistema cardiovascular. Os sinais e sintomas relatados pelos pacientes na chegada ao atendimento de saúde para o procedimento cirúrgico foram: angina $(n=240 ; \quad 69,76 \%), \quad$ Hipertensão Arterial Sistêmica (HAS) $(\mathrm{n}=31 ; 9,01 \%)$, dispneia $(\mathrm{n}=18 ; 5,23 \%)$ e sudorese $(\mathrm{n}=14 ; 4,06 \%)$.

Quanto às doenças preexistentes dos pacientes, ( $\mathrm{n}=144 \mathrm{41,86 \% )}$ portavam pelo menos uma doença, e ( $n=99$ 28,77\%) entre duas e quatro. Metade da amostra apresentava HAS $(\mathrm{n}=187 ; 54,36 \%)$, seguido de DM $(\mathrm{n}=$ $111 ; 32,26 \%)$, dislipidemia $(n=80 ; 23,25 \%)$ e IAM $(n=69 ; 20,05 \%)$, como demonstrado na tabela 02 . 
Tabela 02 - Características clínicas dos pacientes submetidos a ICP entre 2014 e 2019. Uruguaiana, Brasil, 2021

\begin{tabular}{|c|c|c|}
\hline Variável & $\mathrm{N}$ & $\%$ \\
\hline \multicolumn{3}{|l|}{ Doenças } \\
\hline Angina Instável & 110 & 31,97 \\
\hline IAM, não especificado & 107 & 31,10 \\
\hline Angina pectoris, não especificada & 83 & 24,12 \\
\hline Doença isquêmica crônica do coração & 83 & 24,12 \\
\hline Outro & 07 & 2,03 \\
\hline \multicolumn{3}{|l|}{ Sinais e sintomas } \\
\hline Angina & 240 & 69,76 \\
\hline HAS & 31 & 9,01 \\
\hline Dispneia & 18 & 5,23 \\
\hline Sudorese & 14 & 4,06 \\
\hline Náuseas & 12 & 3,48 \\
\hline Síncope & 09 & 2,61 \\
\hline Êmese & 07 & 2,03 \\
\hline \multicolumn{3}{|l|}{ Doenças preexistentes } \\
\hline HAS & 187 & 54,36 \\
\hline DM & 99 & 28,77 \\
\hline Dislipidemia & 80 & 23,25 \\
\hline IAM & 69 & 20,05 \\
\hline DM II & 11 & 3,19 \\
\hline ICC & 03 & 0,87 \\
\hline DM I & 01 & 0,29 \\
\hline IRA & 00 & 0,00 \\
\hline
\end{tabular}

IAM = Infarto Agudo do Miocárdio; HAS = Hipertensão Arterial Sistêmica; DM = Diabetes Mellitus; ICC = Insuficiência Cardíaca Congestiva; IRA = Insuficiência Renal Aguda. Fonte: Os autores

Da amostra, $80(23,25 \%)$ pacientes realizaram cateterismo cardíaco seguido de tratamento cardíaco por meio de angioplastia. Metade da amostra $(n=200 ; 58,13 \%)$ foi submetida apenas à angioplastia com a colocação de um, dois ou três stents (gráfico 01). Quanto ao tipo de stent, em $(n=273$ 79,36\%) pacientes foi implantado o convencional e em $(n=71$ 
20,63\%) o tipo farmacológico. Em relação ao desfecho clínico da internação, (n=331 $96,22 \%$ ) dos pacientes tiveram alta hospitalar, $(\mathrm{n}=072,03 \%)$ transferência do setor, e $(\mathrm{n}=06$ $1,74 \%)$ evoluíram para óbito.

Gráfico 01- Procedimentos realizados no serviço de hemodinâmica entre 2014 e 2019. Uruguaiana, Brasil, 2021

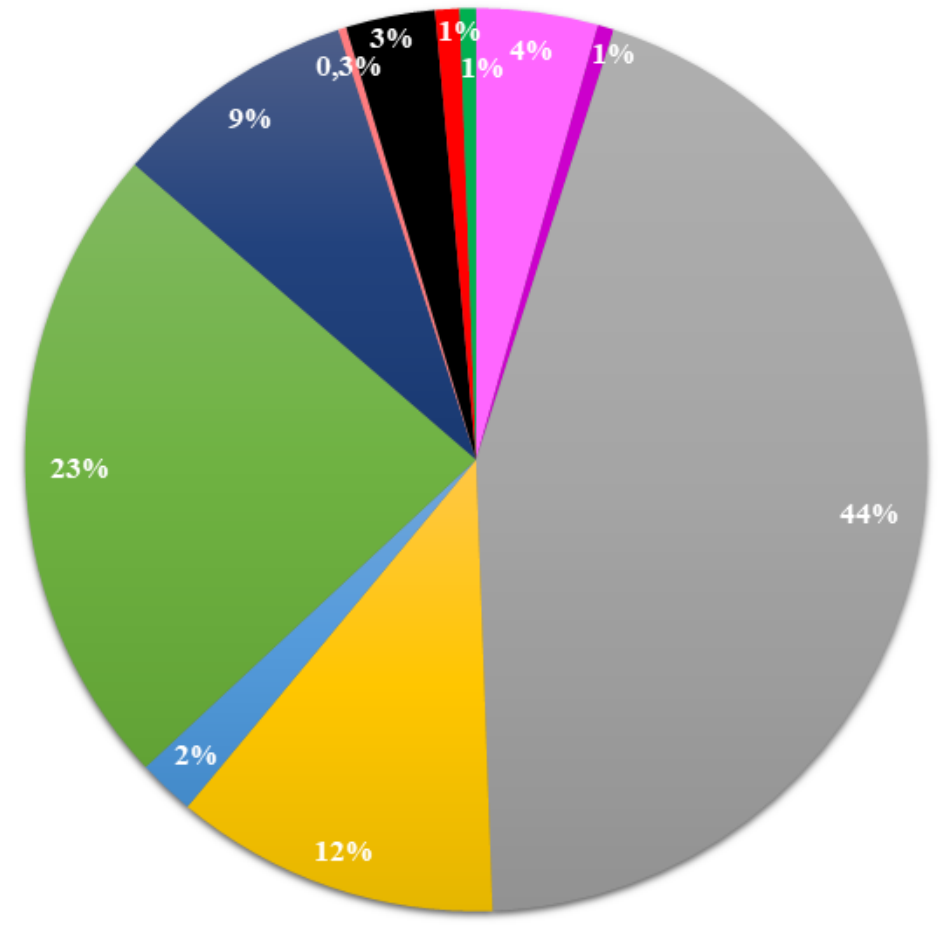

1. Cateterismo

3. Angioplastia com stent

- 5. Angioplastia com três stents ou mais

a 7. Cateterismo e Angioplastia com dois stents

๑. Implante de MP

11. Sem sucesso
-2. Angioplastia sem stent

4. Angioplastia com dois stents

6. Cateterismo e Angioplastia

घ. Cateterismo e Angioplastia com três stents ou mais

10. Outro

Fonte: Dados da pesquisa, 2020

\section{DISCUSSÃO}

Este estudo identificou as características clínicas de pacientes submetidos à intervenção cirúrgica em um serviço de hemodinâmica entre os anos de 2014 e 2019. A média de idade da amostra revela que são idosos jovens com uma doença cardiológica preexistente.

O percentual alto de homens submetidos a ICP em relação às mulheres, pode estar relacionado às construções sociais de gênero que influenciam no modo como percebem e enfrentam os cuidados com a saúde individual. Em geral, a manutenção https://doi.org/10.31011/reaid-2021-v.95-n.36-art.1146 Rev Enferm Atual In Derme v. 95, n. 36, 2021 e-021171 
preventiva e contínua à saúde realizada pela população feminina torna-se um fator protetor em relação à masculina. Este dado fica ainda mais evidente quando identifica-se que mais da metade dos homens foram submetidos à intervenção de urgência, contrapondo ao sexo feminino. Além disso, o número de intervenções eletivas entre as mulheres pode estar atrelado à proteção oriunda do hormônio estradiol até a menopausa ${ }^{(7,8)}$.

A angina foi a característica clínica mais relatada pelo paciente na chegada para atendimento de saúde, demonstrando que a dor na região torácica de diferentes variações de intensidade é capaz de sensibilizar a busca por atendimento emergencial. Ademais, um estudo reforça que a dor torácica é um dos motivos de maior procura pelos serviços de emergência, sendo classificada como insuportável pelos pacientes, levando muitas vezes a dispnéia ${ }^{(9)}$.

A intensidade da dor também pode estar associada a outros sintomas como sudorese, náusea e êmese, os quais corroboram com a busca do paciente no serviço de saúde ${ }^{(10)}$. Fisiologicamente, as DCV podem ocasionar dispnéia e síncope em decorrência da congestão pulmonar e pela diminuição transitória do fluxo cerebral, como evidenciado neste estudo ${ }^{(11)}$.

Outro motivo que conduziu o paciente ao atendimento no serviço de saúde foi a agudização da HAS. A identificação de mais da metade da amostra com uma ou duas doenças prévias, instiga a reflexões sobre a identificação e continuidade das intervenções multidisciplinares da pessoa com doença crônica na APS. A continuidade do cuidado na Rede de Atenção à Saúde (RAS) possibilita a redução dos riscos de agudização das doenças crônicas, desfragmentação do cuidado, implementação da clínica ampliada à saúde e controle social baseado em modelos clínico e epidemiológico.

Dentre as possíveis intervenções que viabilizam a identificação e continuidade da assistência, evidencia-se as ações de promoção de saúde para controle das doenças prévias do Plano de Ações Estratégicas para o Enfrentamento das Doenças Crônicas Não Transmissíveis e a Política Nacional de Atenção Integral à Saúde do Homem. Para as doenças cardiovasculares, as ações que incluem o uso racional de medicamentos aos pacientes portadores de $\mathrm{HAS}^{(12)}$ e portadores de outras doenças crônicas, visto ser um fator protetor para o desenvolvimento e agravamento da DVC e prevenção para outras doenças.

As doenças preexistentes identificadas nesta amostra vêm ao encontro com outro estudo desenvolvido entre os anos de 2000 e 2010, no qual evidenciou uma associação significativa da presença de diabetes $(25,2 \%)$ e HAS $(53,1 \%)$ entre idosos residentes no município de São Paulo ${ }^{(13)}$. Ademais, uma revisão integrativa da literatura evidenciou que a HAS foi o principal fator de risco para o 
IAM, com exceção de um estudo realizado no Chile $^{(14)}$.

Em resposta, o avanço da prática de enfermagem deve incluir ações de promoção, prevenção, tratamento e reabilitação à saúde por meio de tecnologias duras, leve-dura e leve em seu processo de trabalho e cuidado. Essa evidência é identificada na atenção hospitalar, quando os profissionais utilizam equipamentos e instrumentais cirúrgicos específicos para a implantação de stents farmacológicos e convencionais no tratamento das DCV.

A implantação de stents farmacológicos é uma alternativa mais segura e eficaz tendo em vista os diversos resultados indesejáveis do stent convencional, como a hiperplasia neo-intimal, trombose de stent, os quais resultam em um novo IAM e óbito ${ }^{(15)}$. Como este estudo é transversal e não apresenta variáveis sobre as alterações a médio e longo prazo dos pacientes submetidos a tratamento cardiológico no serviço, torna-se inviável sustentar tais complicações clínicas no uso de stent convencional nesta amostra. Contudo, o desfecho clínico da internação revela um percentual aceitável e tolerável para o uso de stent convencional para esta amostra.

Mesmo tendo conhecimento dos benefícios do stent farmacológico identificouse que a maioria utilizou o stent convencional que não apresenta o revestimento medicamentoso. A taxa de revascularização miocárdica é menor naqueles que utilizam o stent farmacológico, porém não há diferença nas taxas de mortalidade quanto ao tipo de stent utilizado ${ }^{(16)}$. Essa evidência é sustentada quando identifica-se que a prevalência para o desfecho clínico da internação, neste estudo, foi a alta hospitalar.

A identificação dos desfechos clínicos, alta hospitalar e óbito, foram semelhantes a outro estudo que evidenciou 95,7\% ( $\mathrm{n}=89)$ para alta hospitalar e 4,3\% (n= 4) para óbito $^{(17)}$. O agravamento que levou ao óbito ocorreu devido a complicações da doença de base, e não do procedimento realizado, pois a angioplastia utilizada como tratamento precoce apresenta baixo risco de complicações $^{(18)}$.

Mediante a alta taxa de alta hospitalar, fica evidente que os pacientes estão retornando para a atenção primária de saúde. É neste momento que a importância da RAS revela-se como essencial na reabilitação destes pacientes. O papel do enfermeiro na atenção secundária pode prever um plano de intervenção voltado à reabilitação cardiovascular, enquanto que o da atenção primária volta-se a reinserir o paciente em seu contexto de vida e saúde. Essa articulação entre os níveis de atenção impulsiona à prática avançada de enfermagem e fortalece a categoria profissional nos diferentes contextos de trabalho.

O baixo percentual de intervenções sem sucesso satisfatório para o tratamento em 
saúde pode, em parte, ser relacionado com as atividades de assistência e gerenciamento adotadas pela equipe de enfermagem. O papel do enfermeiro é essencial em unidades de hemodinâmica, pois abrange a realização de procedimentos de baixa, média e alta complexidade; desenvolver e coordenar ações de educação em saúde; liderar uma equipe multidisciplinar; identificar necessidades de saúde e intervir para preparar o paciente e sua família na readequação das rotinas e hábitos após a alta hospitalar; auxiliar no sucesso da terapêutica, evitando possíveis reinternações e complicações cardíacas $^{(4)}$.

Entretanto, a fragilidade na articulação entre os níveis de atenção reforça a fragmentação do cuidado. Essa constatação também é visível no ambiente hospitalar, pois a ausência de um sistema eletrônico não assegura a continuidade das intervenções realizadas em distintos momentos de (re)internação, alta e/ou transferência do paciente no serviço. Para tal, subentende-se que os pacientes submetidos à angioplastia tenham realizado cateterismo prévio para avaliação diagnóstica do quadro clínico. Ademais, pressupõe-se que os pacientes foram referenciados para serviços de reabilitação cardíaca em seu período de pósoperatório tardio.

A limitação do estudo refere-se a análise retrospectiva em prontuários físicos, os quais conduziu a perdas estatísticas e riscos eminentes de registros incompletos. Sabe-se que o tempo porta-balão do atendimento às emergências cardiológicas é o principal passo para que a intervenção seja eficaz, garantindo a boa recuperação do paciente ${ }^{(19)}$, com isso, outro fator limitante do estudo é a ausência de registros de enfermagem nos prontuários físicos, como por exemplo como o paciente chegou ao atendimento, ou a quanto tempo estava apresentando sintomas, o que impediu a análise aprofundada dos prontuários.

\section{CONCLUSÃO}

Este estudo identificou as características clínicas de pacientes submetidos a ICP, sendo prevalentes o sexo masculino, de cor branca, casados ou com união estável. Quanto ao diagnóstico o mais predominante foi a angina instável, tendo como intervenção mais utilizada a angioplastia com colocação de stent, sendo a maioria do tipo convencional.

O conhecimento acerca do perfil dos pacientes submetidos a ICP é capaz de oportunizar o desenvolvimento de um plano de cuidados ao paciente a ser utilizado na RAS, a fim de articular ações de prevenção, promoção e reabilitação à saúde. Além disso, os resultados deste estudo proporcionam subsídios para otimizar o processo de trabalho e de cuidado da equipe de enfermagem, e principalmente ao enfermeiro, na atenção primária, secundária e terciária, viabilizando uma RAS com qualidade à sociedade. 


\section{REFERÊNCIAS}

1. Figueiredo FSF, Oliveira RR, Sanches RCN, Matias TAF, Radovanovic CAT. Mortalidade por doenças cardiovasculares no estado do paraná. Cogitare Enferm. 2018 [citado 2021 abr 28];(23)4:e56973. DOI: http://dx.doi.org/10.5380/ce.v23i4.56973

2. Teixeira TRF, Avila MAG, Braga EM. Compreensão de pacientes às orientações de enfermagem no cateterismo cardíaco: uma pesquisa qualitativa. Cogitare enferm. 2019 [citado 2021 fev 11];24:e56604. DOI: dx.doi.org/10.5380/ce.v24i0.56604

3. Vicente MRF, Alminhas SMP. Protocolo de desinsuflação de dispositivo de compressão radial (tr band) abreviado após procedimento de cateterismo cardíaco. In: Lobão $C$, Alminhas S, Ferreira N. V Encontro dos Enfermeiros Especialistas em Enfermagem Médico-Cirúrgica.[E-book on the Internet] Ordem dos Enfermeiros; 2018 [citado 2021 mar 04]. p.40-41. Disponível em:

https://www.ordemenfermeiros.pt/media/8860 /livro-de-resumos-v-encontroevora_vfinalissima-003.pdf\#page $=40$

4. Rodrigues MEA, Lopes GS, Souza LA, Bié ALA, Colares LDS, Souza ACO, et al. Angioplastia Coronária: adversdades e possibilidades na assistência de enfermagem. BJHR. 2021 [citado 2021 mar 03];4(1):234766. DOI: $10.34119 /$ bjhrv4n1-189

5. Lemos IMN, Paixão IMS, Silva IIM, Oliveira ERJ, Cerqueira LMR. O papel do enfermeiro dentro da unidade de hemodinâmica. International Nursing Congress. 2017 [citado 14 jul 2021]; 1(1): 9-12. Disponível em: https://eventos.set.edu.br/cie/article/view/590 $\underline{0 / 2201}$

6. Régis AP, Rosa GCD, Lunelli T. Cuidados de enfermagem no cateterismo cardíaco e angioplastia coronariana: desenvolvimento de um instrumento. Recien. 2017 [citado 14 jul 2021]; 7(21):3-20. Disponível em: https://www.recien.com.br/index.php/Recien/ article/view/238/325

7. Lima MSM, Dantas RAN, Mendes NPN, Alves LCM, Silva TTM, Brito AGR, et al. Aspectos clínico-epidemiológicos de pacientes sometidos a la Intervención Coronaria Percutánea en un hospital universitario. Rev. Bras. Enferm. 2018 [citado 2021 fev 11];71(6)2883-90. DOI: https://doi.org/10.1590/0034-7167-2018-0012

8. Costa FAS, Pessoa VLMP, Frota KC, Araújo DV, Almeida VS. Aspectos clínicoepidemiológicos de pacientes com infarto agudo do miocárdio submetidos à angioplastia coronariana primária. Essentia. 2020 [citado 2021 abr 23];21(1):21-6. DOI: https://doi.org/10.36977/ercct.v21i1.332

9. Figueiredo Júnior AM, Galvão MM, Souza JP. Percepções da dor: diagnóstico de enfermagem em pacientes infartados. REAS/EJCH. 2019 [citado 2021 abr 28]; $21 \mathrm{e} 547$.

DOI:

https://doi.org/10.25248/reas.e547.2019

10. Souza CFQ, Souza SM, Batista RPS, Bandeira TMP, Neto WB, Silva Junior WS. Aplicativo móvel como ferramenta de assistência e prevenção ao infarto agudo do miocárdio. Enfermería Actual de Costa Rica. 2020 [citado 2021 fev 27];(39):129-143. DOI:

http://dx.doi.org/10.15517/revenf.v0i39.4107 2.

11. Porto AL. Anamnese - Sequência racional. In: Silva PGMB, Lopes RD, Lopes AC. Semiologia cardiovascular baseada em evidências.[Internet] Rio de Janeiro: Editora Atheneu; 2018 [citado 2021 mai 02]. p. 1117.

12. Portela PP, Mussi FC, Gama GGG, Santos CAST. Fatores associados ao descontrole da pressão arterial em homens. Acta paul. enferm. 2016 [citado $2021 \quad$ Fev 20];29(3):307-315. Doi http://dx.doi.org/10.1590/19820194201600043.

13. Massa KHC, Duarte YAO, Chiavegatto ADP. Análise da prevalência de doenças cardiovasculares e fatores associados em idosos, 2000-2010. Ciênc. saúde coletiva. 2019 [citado 2021 mar 04];24(1):105-114. DOI: $\quad$ https://doi.org/10.1590/141381232018241.02072017 
14. Pinheiro RHO, Lenhani BE, Martins EV. Prevalência de fatores de risco relacionados ao infarto agudo do miocárdio em pacientes idosos: uma revisão integrativa. Uningá Review. 2017 [citado 2021 abr 28];30(3):8388. Disponível em: http://34.233.57.254/index.php/uningareviews /article/view/2023/1616

15. Ferreira BWRC, Gusmão AB, Duarte LSM, Coutinho MB, Wanderley KG, Macedo CL. Gerações de stents no tratamento da cardiopatia isquêmica. Research, Society and Development. 2020 [citado 2021 abr 14];9(9):e89997049. DOI: http://dx.doi.org/10.33448/rsd-v9i9.7049

16. Ferreira FMR. Comparação entre stent farmacológico e stent convencional na doença arterial coronária: metanálise [dissertação]. Rio de Janeiro: Avaliação de Tecnologias em Saúde do Instituto Nacional de Cardiologia; 2018. [citado 2021 fev 27]. Disponível em: http://200.20.108.73:8080/xmlui/bitstream/ha ndle/374/98/Felipe\%20Miranda\%20\%20ATS.pdf? sequence $=1 \&$ is Allowed $=\mathrm{y}$

17. Santos ACP, Ambiel MLB, Ferreira EB, Rocha PRS. Complicações vasculares e fatores relacionados a sua ocorrência após procedimentos hemodinâmicos percutâneos. Rev. Enferm. UFSM - REUFSM. 2020 [citado 2021 abr 23];10:e90:1-16. DOI: $10.5902 / 2179769241286$

18. Alves GACD, Silva WA, Andrade AN, Ribeiro AGF. Os benefícios da angioplastia nas primeiras horas do infarto agudo do miocárdio com supradesnivelamento do segmento st. Revista Interdisciplinar em Saúde. 2018 [citado 2021 fev 18];5(4):641$54 . \quad$ Disponível em: http://www.interdisciplinaremsaude.com.br/V olume_20/Trabalho_02.pdf

19. Moraes CLK, Tessmann M, Botelho RV, Mendonça V, Schmitt A, Ficagna FT. Perfil e tempo porta-balão de pacientes com infarto agudo do miocárdio. Revista Inova Saúde. 2020 [citado 2021 fev 18];10(2). DOI: http://dx.doi.org/10.18616/inova.v10i2.5320
Submissão: 2021-06-21

Aprovado: 2021-09-02 
Anexo A

Parecer Consubstanciado do CEP

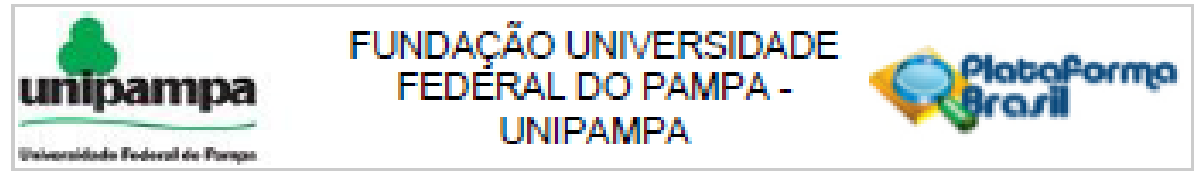

\section{PARECER CONSUBSTANCIADO DO CEP}

DADOS DO PRONETO DE PESQUISA

Titulo da Pocqules: Caracteristicas clinicas dos Paclentes aubmetidos a Intervenç5o Coronariana Percutanea no aerviço de Hemodinamica

Pocquicador: Letce Dala Lana

Area Tematioa:

Vercso: 2

CAAE: 30498320.0 .0000 .5323

Inctitulọso Proponente: Fundoģ5o Univeraldade Federal do Fampa UNIFAMPA

Patroolnador Prinolpal: Financiamento Proprio

DADOS DO PARECER

Numero do Parecer: 4.157.649

Aprocentagso do Projoto:

As doenças cardlovasculares acometem grande parte da populaçbo brasleira, demandando de intervençbes Imedlatas e emergenciala. Dentre as principala Intervençbes, destacam-se a Anglografla ou a Angloplasta Coronaria Percutanea Transluminal (FTCA). Acreditando na importancla deates procedimentos e na resolublidade gerada aos pacientes cardlopatas, este estudo visa identiflcar as caracteriaticas cilnicas dos pacientes submetidoa a intervençbo Coronariana Percutanea (ICP) atendidos no serviço de Hemodinamica do Hoaplaj Banta Casa de aaude de Unugualana. Metodo: Extudo retroapectivo, quanttativo e documental a ser desenvolvido na hemodinamica de um hospital da regl5o sudeste da fronteira. A amostra sera de 370 prontuarios dlstrbulbos entre os 8 anos de funcionaldade do serviço. A anallse dos dados sera eatatistica aimples. Resultados eaperados: eapera-se que a identficaç5o do perfll de pacientes tranacreva a importancla e a reievancla de de"inir um fluxo de atendimento so paciente cardlopata. Acredlta-se ainda que oa dados, podem contribulr para o desenvolvimento de eatrattglas de prevençlo de fatores de riaco para doenças arterials coronarianas e, nesse sentido, viabllizar a construçbo de habllidades e competenclas a equpe de saude, em eapecial a atuaç\$o da equje de enfermagem.

aitusgso do Parooer:

Aprovado

Noceccita Apreologso da CONEP:

NSo

URUGUALANA, 16 de Juho de 2020

Acclinado por:

Jullana Lopec de Maoedo

[Coordensdor $(a)]$

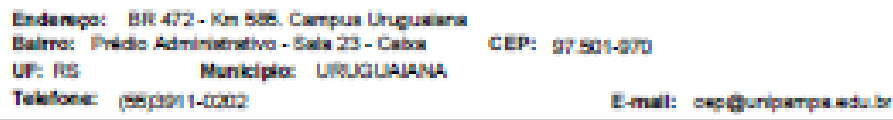




\section{Apêndice A}

Serviço de Hemodinâmica

INSTRUMENTO DE COLETA DE DADOS - ANÁLISE DOCUMENTAL: PRONTUÁRIO DO PACIENTE

Instrumento $\mathrm{N}^{\circ}$ : Data da coleta dos dados:

Responsável pela coleta:

\section{CARACTERÍSTICAS CLÍNICAS}

1.1 Gênero: (1)Feminino (2)Masculino 1.2 Idade:____ anos

1.3 Cor/Raça: (1)Branco (2)Negro (3)Pardo (4)Outro:

1.4 Bairro que reside:

1.5 Data do procedimento _____

1.6 Tipo de procedimento PROPOSTO: REALIZADO

1.7 Nacionalidade: (1)Brasileiro (2)Argentino (3)Uruguaio (4)Outros:

1.8 Naturalidade: (1)Uruguaiana (2)Itaqui (3)Barra do Quaraí (4)Alegrete (5)São Borja (6)Quaraí (7)Santana do Livramento (8)Maçambará (9)Garruchos (10)Rosário do Sul (11)São Gabriel (12)Manoel Viana (13)Itacurubi (14)Santa Margarida do Sul (15)Outros:

1.9 Estado civil: (1)Solteiro (2)Casado (3)União estável (4)Divorciado (5)Viúvo (6)Outro:

1.10 Escolaridade: (1)Analfabeto (2)Ensino Fundamental Incompleto (3)Ensino Fundamental Completo (4)Ensino Médio Incompleto (5)Ensino Médio Completo (6)Ensino Superior Completo (7)Ensino Superior Incompleto

1.11 Profissão/ocupação: (1)Aposentado (2) Autonomo (3) Desempregado/Do Lar (4)Trabalhador doméstico (5) Professor(a) (6) Profissional da saúde (7)Agricultor (8)Funcionário Público (9)Comerciante (10)Atendente/vendedor (12)Advogado (5)Outro:

\section{CARACTERÍSTICAS CLÍNICAS}

2.1 CID e/ou diagnóstico no momento da internação na hemodinâmica:

2.2 Sinais e sintomas no momento da internação: 
2.3 Doenças preexistente:

2.3.1 IAM: (1) Sim; (2) Não; (3) Dado não disponível (4) Dado inelegível 2.3.2 HAS: (1) Sim; (2) Não; (3) Dado não disponível (4) Dado inelegível 2.3.3 DM: (1) Sim; (2) Não; (3) Dado não disponível (4) Dado inelegível 2.3.4 DM I: (1) Sim; (2) Não; (3) Dado não disponível (4) Dado inelegível 2.3.5 DMII:(1) Sim; (2) Não; (3) Dado não disponível (4) Dado inelegível 2.3.6 IRA: (1) Sim; (2) Não; (3) Dado não disponível (4) Dado inelegível 2.3.7 ICC: (1) Sim; (2) Não; (3) Dado não disponível (4) Dado inelegível 2.3.8 outras:

2.4 Fatores de risco modificáveis:

2.4.1 Tabagismo: (1) Sim; (2) Não; (3) Dado não disponível (4) Dado inelegível 2.4.2 Etilismo: (1) Sim; (2) Não; (3) Dado não disponível (4) Dado inelegível 2.4.3 Obesidade: (1) Sim; (2) Não; (3) Dado não disponível (4) Dado inelegível 2.4.4 Sedentarismo: (1) Sim; (2) Não; (3) Dado não disponível (4) Dado inelegível 2.4.5 Dislipidemia: (1) Sim; (2) Não; (3) Dado não disponível (4) Dado inelegível 2.4.6 Drogas Ilícitas: (1) Sim; (2) Não; (3) Dado não disponível (4) Dado inelegível 2.4.7 Alimentação inadequada: (1) Sim; (2) Não; (3) Dado não disponível (4) Dado inelegível 2.5 Desfecho clínico:

2.5.1(1)Transferência de setor (2)Alta da Unidade (3)Óbito 2.5.2 intervenção prévia: 